\title{
Myeloid cell leukemia-1 is an important apoptotic survival factor in triple-negative breast cancer
}

\author{
CM Goodwin ${ }^{1}$, OW Rossanese ${ }^{2,3}$, ET Olejniczak ${ }^{2,3}$ and SW Fesik ${ }^{\star, 1,2,3}$
}

Breast cancer is the second-most frequently diagnosed malignancy in US women. The triple-negative breast cancer (TNBC) subtype, which lacks expression of the estrogen receptor, progesterone receptor and human epidermal growth factor receptor-2, afflicts $15 \%$ of patients and is refractory to current targeted therapies. Like many cancers, TNBC cells often deregulate programmed cell death by upregulating anti-apoptotic proteins of the B-cell CLL/lymphoma 2 (Bcl-2) family. One family member, myeloid cell leukemia-1 (Mcl-1), is commonly amplified in TNBC and correlates with a poor clinical prognosis. Here we show the effect of silencing Mcl-1 and Bcl-2-like protein 1 isoform 1 (Bcl-xL) expression on viability in a panel of seventeen TNBC cell lines. Cell death was observed in a subset upon Mcl-1 knockdown. In contrast, Bcl-xL knockdown only modestly reduced viability, indicating that Mcl-1 is a more important survival factor. However, dual silencing of both Mcl-1 and Bcl-xL reduced viability in most cell lines tested. These proliferation results were recapitulated by BH3 profiling experiments. Treatment with a Bcl-xL and Bcl-2 peptide had only a moderate effect on any of the TNBC cell lines, however, co-dosing an Mcl-1-selective peptide with a peptide that inhibits Bcl-xL and Bcl-2 was effective in each line tested. Similarly, the selective Bcl-xL inhibitor WEHI-539 was only weakly cytotoxic across the panel, but sensitization by Mcl-1 knockdown markedly improved its EC $_{50}$. ABT-199, which selectively inhibits $\mathrm{Bcl}-2$, did not synergize with Mcl-1 knockdown, indicating the relatively low importance of Bcl-2 in these lines. Mcl-1 sensitivity is not predicted by mRNA or protein levels of a single Bcl-2 family member, except for only a weak correlation for Bak and Bax protein expression. However, a more comprehensive index composed of Mcl-1, Bcl-xL, Bim, Bak and Noxa protein or mRNA expression correlates well with Mcl-1 sensitivity in TNBC and can also predict Mcl-1 dependency in non-small cell lung cancer cell lines. Cell Death and Differentiation (2015) 22, 2098-2106; doi:10.1038/cdd.2015.73; published online 5 May 2015

Breast cancer is the second-most frequently diagnosed malignancy in US women with 230000 new cases and 40000 deaths in 2011. The triple-negative breast carcinoma (TNBC) subtype, which does not express the estrogen receptor (ER) and progesterone receptor (PR) and lacks overexpression of human epidermal growth factor receptor-2 (HER2), afflicts nearly $15 \%$ of all breast cancer patients and remains refractory to currently available endocrine and HER2directed therapies. ${ }^{1,2}$ The current standard of care for TNBC is radiation and neoadjuvant cytotoxic chemotherapy, and carries a poor clinical prognosis. ${ }^{3-5}$

As with most cancers, TNBC cells are under metabolic and oncogenic stress and require inhibition of the intrinsic apoptotic pathway for survival. ${ }^{6}$ Under normal physiological conditions, this pathway is tightly regulated by both pro- and anti-apoptotic members of the B-cell CLL/lymphoma 2 (Bcl-2) family. Stressors such as DNA damage, hypoxia or oncogenic signaling, cause increased expression or translocation of proapoptotic Bcl-2 family members, such as Bim, Bad and Noxa, to the mitochondria. ${ }^{7}$ These proteins subsequently trigger pore formation in the mitochondrial outer membrane via induced multimerization of Bak or Bax, a process that leads to cytochrome $\mathrm{c}$ release, caspase cleavage and commitment to apoptosis.

In the absence of environmental or oncogenic stressors, multi-domain Bcl-2 family members such as myeloid cell leukemia-1 (Mcl-1), Bcl-2 and Bcl-2-like protein 1 isoform 1 (Bcl-xL), prevent apoptosis by sequestering the pro-apoptotic family members. Many cancer types aberrantly block oncogenic apoptotic signaling by increasing steady-state expression of one or more of these proteins through genetic amplification, transcript upregulation or reduced degradation. ${ }^{8}$ It is therefore unsurprising that Bcl-2 family inhibitors, such as ABT-263 and ABT-199, have displayed pre-clinical and clinical efficacy. ${ }^{9-11}$ Effectively using these targeted therapeutics requires accurately predicting which anti-apoptotic proteins the tumor depends upon for survival. High expression of a pro-survival Bcl-2 family member does not necessarily correlate with dependency on that protein to prevent apoptosis. ${ }^{12-14}$ Individual pro-survival $\mathrm{Bcl}-2$ family proteins preferentially inhibit a subset of pro-apoptotic family members, ${ }^{15}$ and cancer cells require a counterbalancing antagonist for whichever proapoptotic stimuli are present. Moreover, additional regulatory mechanisms, such as limiting trafficking to the mitochondria or

${ }^{1}$ Department of Pharmacology, Vanderbilt University School of Medicine, Nashville, TN, USA; ${ }^{2}$ Department of Biochemistry, Vanderbilt University School of Medicine, Nashville, TN, USA and ${ }^{3}$ Vanderbilt-Ingram Cancer Center, Vanderbilt University School of Medicine, Nashville, TN, USA

${ }^{*}$ Corresponding author: SW Fesik, Department of Biochemistry, Vanderbilt University School of Medicine, 2215 Garland Avenue, 607 Light Hall, Nashville, TN 372320146 , USA. Tel: +1 615322 6303; Fax: +1 615875 3236; E-mail: stephen.fesik@vanderbilt.edu

Abbreviations: Mcl-1, myeloid cell leukemia-2; TNBC, triple-negative breast cancer; ER, estrogen receptor; PR, progesterone receptor; HER2, human epidermal growth factor receptor-2; Bcl-2, B-cell CLL/lymphoma 2; Bcl-xL, Bcl-2-like protein 1 isoform 1; BH3, Bcl-2 homology domain 3; siRNA, small-interfering RNA; NSC, non-silencing control oligonucleotides; CCLE, Broad-Novartis Cancer Cell Line Encyclopedia.

Received 30.12.14; revised 16.4.15; accepted 28.4.15; Edited by G Melino; published online 05.5.15 
inducing degradation, may alter activity regardless of the absolute protein level expressed. ${ }^{16}$ More accurate predictions use a multi-protein index, such as the ratio of $\mathrm{Mcl}-1$ to $\mathrm{Bcl}-\mathrm{xL}$ to predict $\mathrm{Mcl}-1$ dependency in small cell lung carcinoma, ${ }^{17}$ or the ratio of phospho-Bcl-2/(Mcl-1+Bcl-2) to predict sensitivity to the pan-Bcl-2 inhibitor S1 in leukemia. ${ }^{18}$

$\mathrm{Mcl}-1$ overexpression has been reported in several hematological and solid tumor cancers, and is one of most frequently amplified genes in human cancer ${ }^{19,20}$ including prostate, lung, pancreatic, breast, ovarian, melanoma, B-cell chronic lymphocytic leukemia, acute myeloid leukemia and acute lymphoblastic leukemia. Overexpression in breast cancer is associated with a high tumor grade and poor survival, ${ }^{21}$ and pre-clinical evidence suggests that Mcl-1 represents a promising target for the treatment of breast cancers. $^{19,22,23}$ Indeed, the MCL1 gene is the most common genetic amplification (after TP53) that occurs following neoadjuvent therapy in TNBC. ${ }^{24}$ Further, Mcl-1 overexpression is implicated as a resistance factor for multiple therapies, including the widely prescribed microtubule-targeted agents paclitaxel and vincristine, ${ }^{25}$ and compounds that inhibit the related family members $\mathrm{Bcl}-2$ and $\mathrm{Bcl}-\mathrm{xL}^{26,27}$ Doxycyclineinducible overexpression of the $\mathrm{Mcl}-1$ antagonizing $\mathrm{Bcl}-2$ homology domain 3 (BH3)-only protein Noxa, but not Bim, Puma or tBid, synergizes with $\mathrm{Bcl}-2$ and $\mathrm{Bcl}-\mathrm{xL}$ inhibitor ABT-737 treatment in Jurkat J16 cells. ${ }^{28}$ The forced overexpression of $\mathrm{Mcl}-1$ in transgenic mice has been reported to exhibit a high incidence of B-cell lymphoma, ${ }^{29}$ whereas $\mathrm{Mcl}-1$ downregulation using antisense oligonucleotides or smallinterfering RNA (siRNA) has been shown to induce apoptosis in a number of cancer cell types. ${ }^{30,31}$

Similarly, Bcl-xL is commonly amplified in cancer ${ }^{20}$ and has been implicated in preventing various cancers from undergoing programmed cell death, including melanoma ${ }^{32}$ and colon cancer stem cells. ${ }^{33}$ In addition, in breast and lung tumors, Bcl-xL amplification correlates with a decreased sensitivity to transcriptional repressors. ${ }^{19}$

Here we explore $\mathrm{Mcl}-1$ and $\mathrm{Bcl}-\mathrm{xL}$ dependencies in a panel of TNBC cell lines and investigate the apoptotic proteins that principally determine sensitivity. These data will help identify the genetic and molecular determinants of sensitivity to antiapoptotic inhibitors in TNBC, which could aid in the design of clinical trials of $\mathrm{Mcl}-1$ inhibitors, patient selection and biomarker identification.

\section{Results}

Mcl-1 protects a subset of TNBC cell lines from apoptosis. As $\mathrm{Mcl}-1$ and $\mathrm{Bcl}-\mathrm{xL}$ are frequently upregulated in human breast cancer, and based on their importance to prevent apoptosis in many cancer types, we hypothesized that some TNBC cell lines may depend on these proteins to prevent apoptosis, and that silencing one or both would reduce cell viability. To test this hypothesis, we silenced the expression of $\mathrm{Mcl}-1$ or $\mathrm{Bcl}-\mathrm{xL}$ using siRNAs. We first validated our siRNA protocol: of the four unique motifs tested for each protein, Mcl-1 motif-17 and Bcl-xL motif-14 reproducibly yielded the greatest knockdown of over $85 \%$ compared with non-silencing control (NSC) in MDA-MB-468 and MDA-MB-453 cells (Supplementary Figures S1A and B). The NSC motif did not lower cell viability; however, silencing Mcl-1 expression substantially reduced the viability of MDAMB-468 cells in a protein knockdown-dependent manner (Supplementary Figures S1C and D). In contrast, silencing $\mathrm{Bcl}-\mathrm{xL}$ had a smaller effect on both cell lines despite high protein knockdown (>90\%).

We silenced $\mathrm{Mcl}-1$ or $\mathrm{Bcl}-\mathrm{xL}$ using these siRNAs in a panel of 17 publicly available luminal and basal cell lines (Figure 1). Cell viability was measured after 5 days to allow for several population doublings, and knockdown efficiency was verified by western blot at the conclusion of the experiment (Supplementary Table S1). Mcl-1 knockdown markedly reduced proliferation by $60 \%$ or more in seven lines (blue, Figure 1a). Four lines have an intermediate dependency on Mcl-1 (40-60\% loss, cyan), and the remainder were largely insensitive (red). We observed increased caspase 3/7 activity after Mcl-1 siRNA transfection only in Mcl-1-dependent, but not independent, cell lines (Figure 1b, Supplementary Figure S2). Similarly, using flow cytometry we observed an increase in apoptotic marker Annexin V staining after Mcl-1 knockdown only in dependent cell lines (Supplementary Figure S3). These data suggest that a subset of our cell lines are solely dependent on Mcl-1 for survival, and reduced proliferation after $\mathrm{Mcl}-1$ protein knockdown is caused by apoptotic induction.

In contrast, siRNA silencing of Bcl-xL expression had only a modest effect on viability for most of the cell lines tested (Figure 1c). Only 2 of the 17 lines qualify as sensitive under our criteria, one of which is also sensitive to Mcl-1 silencing (HCC1395). These data suggest that unlike Mcl-1, few of these TNBC lines depend solely on Bcl-xL for survival.

It is easy to envision that some TNBC cell lines may block apoptosis via protection from two or more pro-survival Bcl-2 family members. To test this, we silenced the expression of both $\mathrm{Mcl}-1$ and $\mathrm{Bcl}-\mathrm{xL}$ proteins with concurrent siRNA treatment. Knockdown of both proteins results in significantly more cell death across the TNBC panel than with knockdown of either protein alone (Figure 1d), including for cell lines insensitive to Mcl-1 knockdown.

BH3 profiling confirms the specific dependency on Mcl-1 for survival. $\mathrm{BH} 3$ profiling has been used to predict the sensitivity of cell lines and patient-derived hematologic cancer cells to Bcl-2 family inhibitors and to define the dependence on specific family members. ${ }^{34,35}$ This assay quantifies mitochondrial depolarization after administration of a BH3-derived peptide in digitonin-permeabilized whole cells. These peptides have differing binding specificities to Bcl-2 family members, which allows for a sophisticated dissection of the individual contributions of these proteins to prevent apoptosis.

We performed $\mathrm{BH} 3$ profiling on a subset of our TNBC panel to further elucidate the protective role of individual antiapoptotic Bcl-2 family members. Treatment with $10 \mu \mathrm{M}$ of the highly potent and pan-Bcl-2 family antagonist Bim-BH3 peptide depolarized the mitochondria in all cell lines tested (Figure 2a), verifying that they are sensitive to BH3-mediated antagonism. In contrast, treatment with the Mcl-1-specific peptide MS-1 (Figure $2 \mathrm{~b})^{36}$ at $100 \mu \mathrm{M}$ depolarized mitochondria only in the $\mathrm{Mcl}-1$-dependent cell lines. The differing concentrations are used to reflect the lower activity of MS-1 
a

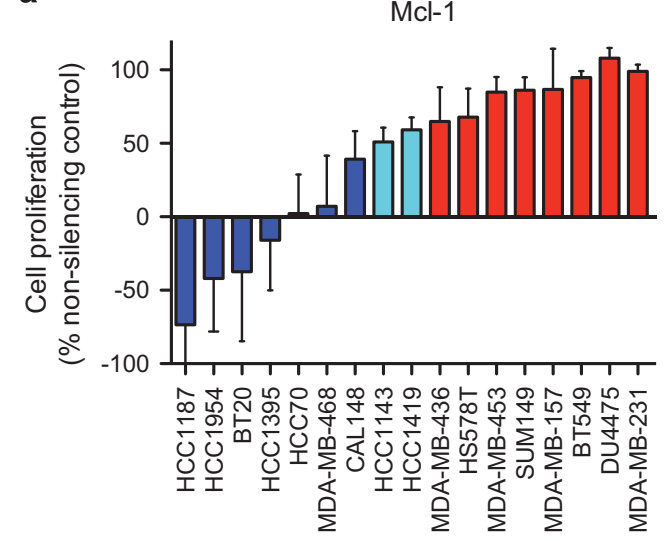

b

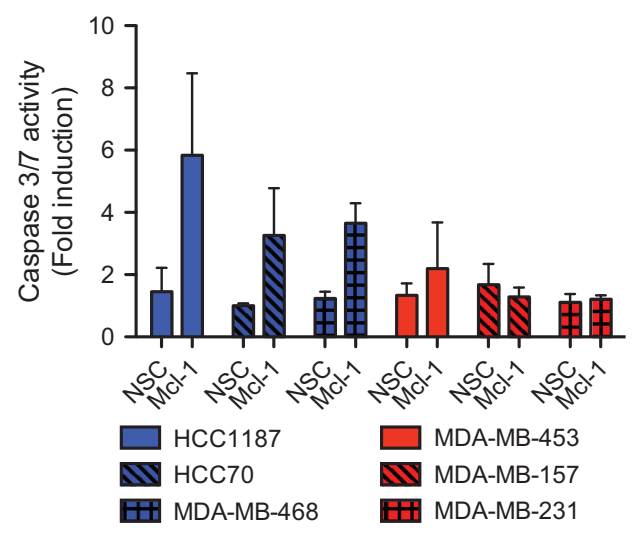

d

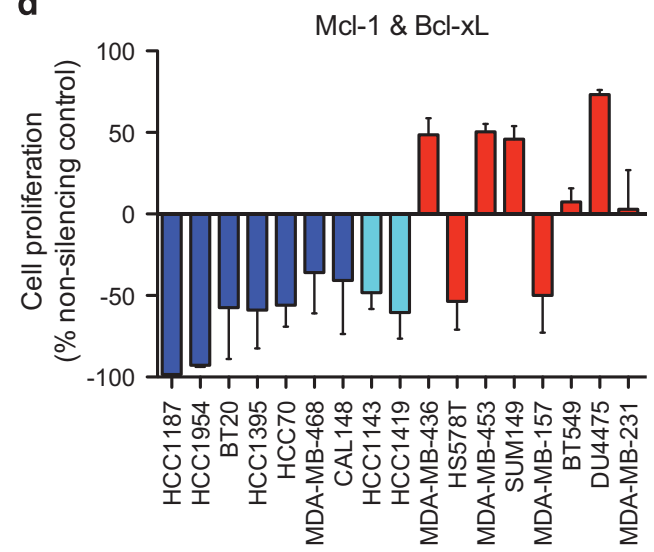

C

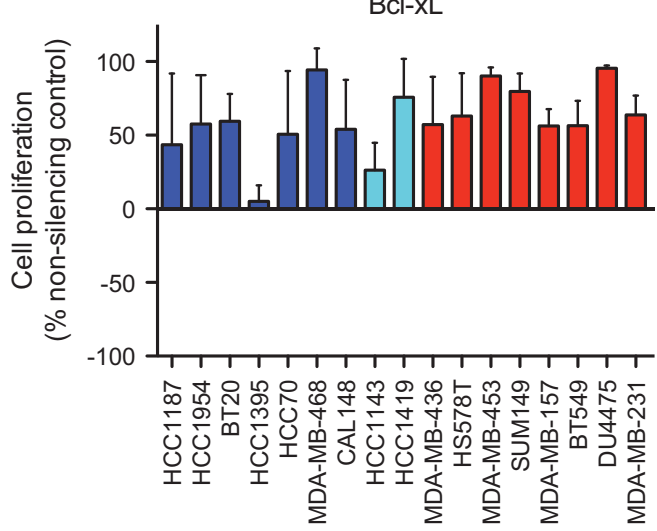

Figure 1 A subset of TNBC cell lines are solely dependent on Mcl-1 to evade apoptosis. (a) Mcl-1 expression was silenced by siRNA and cell proliferation normalized as the ratio of siRNA-treated to NSC-treated cells, subtracting each value by the initial cell count. With this scaling, $100 \%$ indicates knockdown has no effect on viability compared with NSC, $0 \%$ means no change in cell count and negative values indicate cell death. A subset of lines are sensitive to Mcl- 1 knockdown (cell viability reduced by $60 \%$ or more, blue). Two lines are intermediately sensitive (40-60\% reduced viability, cyan), whereas the remainder are Mcl- 1 loss insensitive (red). (b) Fold-induction of caspase $3 / 7$ activity from untreated cells as measured by Caspase-Glo. Mcl-1 knockdown but not NSC siRNA induces caspase 3/7 activity in dependent, but not independent, cell lines after $24 \mathrm{~h}$. (c) Bcl-xL expression was silenced by siRNA. Two lines are sensitive with $>60 \%$ reduction in proliferation. In contrast, dually silencing Mcl- 1 and Bcl-xL expression (d) reduces viability in most lines. Values are the mean of at least three independent experiments (error bars S.D.)

and are consistent with prior concentrations used for this peptide and others in the literature. ${ }^{36}$ The Bad-BH3 peptide, which antagonizes $\mathrm{Bcl}-2$ and $\mathrm{Bcl}-\mathrm{w}$ in addition to Bcl-xL, depolarized mitochondria to a lesser extent than did the MS-1 peptide, which also agrees with the Bcl-xL siRNA results above (Figure 2c). Of note is the higher-than-expected response to Bad-BH3 in MDA-MB-231 cells, although this is perhaps explained by the broad activity of Bad-BH3 to also antagonize other Bcl-2 family members.

Finally, we simulated our dual siRNA results by co-administering the MS-1 peptide and a sub-E10 dose $(50 \mathrm{nM})$ of Bad-BH3, a concentration chosen to clearly demonstrate the synergistic effect of co-dosing. In agreement with our previous results, the mitochondria depolarization increased compared with MS-1 peptide alone for the three Mcl-1-independent cell lines tested. The change in polarization for MDA-MB-453 exceeds that expected from our dual siRNA experiments, presumably because of the expanded binding profile of Bad-BH3. Not surprisingly, increasing the concentration of Bad-BH3 peptide to $1 \mu \mathrm{M}$ further sensitized these cell lines to MS-1 peptide (data not shown).
Mcl-1 knockdown overcomes resistance to Bcl-xL smallmolecule inhibitors. As cell lines in our TNBC panel are largely resistant to $\mathrm{Bcl}-\mathrm{xL}$ silencing, we reasoned these lines may also be resistant to the Bcl-xL-specific small-molecule inhibitor WEHI-539, ${ }^{37}$ and that co-dosing with Mcl-1 knockdown may synergistically reduce viability. To test this hypothesis, we measured the viability $\mathrm{IC}_{50}$ for WEHI-539 during concurrent treatment by NSC or Mcl-1 siRNA (Figure $3 \mathrm{a}$ ). $\mathrm{IC}_{50}$ values for NSC-treated cells are in the low micromolar range, indicating that these cells are not highly sensitive based on the potency observed for other Bcl-2 family inhibitors. ${ }^{38}$ However, in combination with Mcl-1 knockdown, compound potency improves markedly across the panel, such as the 15-fold improvement for MDA-MB-157 cells (Figure 3b). We next tested whether $\mathrm{Bcl}-2$ is an important survival factor by repeating the above experiment using Bcl-2-specific inhibitor ABT-199. ${ }^{39}$ Cells were insensitive to compound alone or in combination with Mcl-1 siRNA (Figures $3 \mathrm{c}$ and d). Finally, we repeated this experiment with ABT-263, a Bcl-2 and Bcl-xL dual inhibitor (Figure 3c). ${ }^{30}$ As seen with WEHI-539, cells were largely resistant to 
a

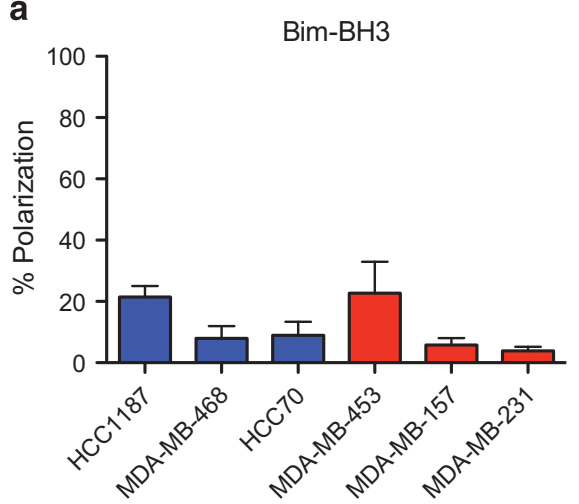

C

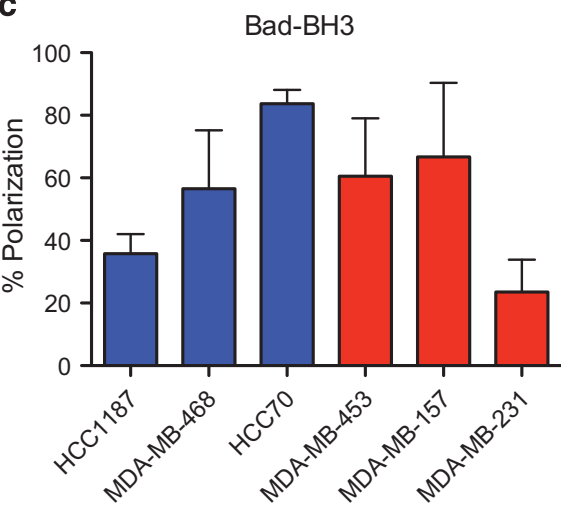

b

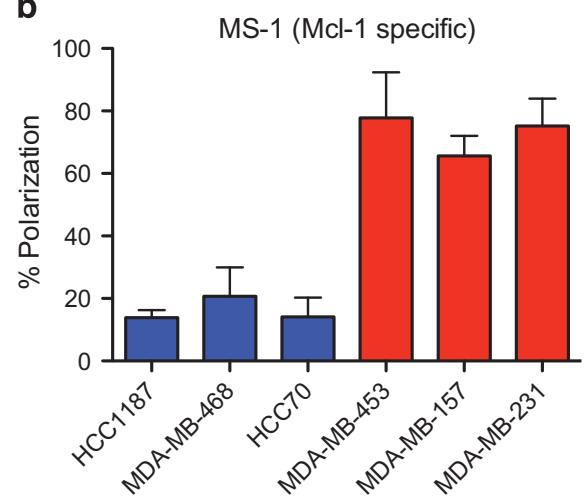

d

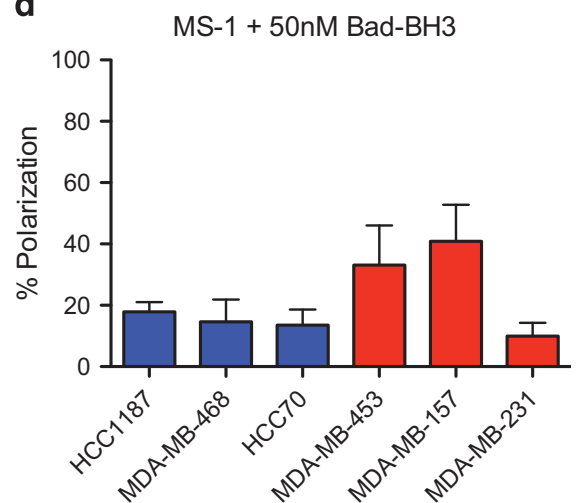

Figure 2 BH3 profiling confirms Mcl-1 dependency status. (a) Whole-cell treatment with $10 \mu \mathrm{M} \mathrm{Bim-BH3} \mathrm{peptide,} \mathrm{a} \mathrm{nonspecific} \mathrm{pro-survival} \mathrm{Bcl-2} \mathrm{family} \mathrm{antagonist,} \mathrm{induced}$ mitochondria depolarization in all cell lines tested. (b) In contrast, the Mcl-1-specific antagonist MS-1 at $100 \mu \mathrm{M}$ selectively depolarized mitochondria only in Mcl-1-dependent cell lines. (c) The Bad-BH3 peptide, which antagonizes Bcl-2 and Bcl-w in addition to Bcl-xL, depolarized mitochondria at $10 \mu \mathrm{M}$ strongly only in the MDA-MB-231 cell line. (d) Combined administration of $0.05 \mu \mathrm{M}$ Bad-BH3 peptide with MS-1 peptide as in a depolarized mitochondria more strongly. Shown are the mean of at least three independent experiments (error bars S.D.)

compound alone but were extremely sensitive to combination with $\mathrm{Mcl}-1$ silencing (Figures $3 e$ and $\mathrm{f}$ ). In summary, the Bcl-xL inhibitors ABT-263 and WEHI-539, but not the selective Bcl-2 inhibitor ABT-199, have an additive effect with Mcl-1 knockdown to reduce cell viability in these cell lines.

Bcl-2 family member mRNA or protein levels do not predict Mcl-1 sensitivity. Our siRNA, compound treatment, and $\mathrm{BH} 3$ profiling experiments all suggest a critical role for Mcl-1 to protect a subset of TNBC lines from apoptosis. However, it was unclear why some (but not all) lines depended on $\mathrm{Mcl}-1$ for survival. We found no correlation between mRNA expression and cell viability in these TNBC lines for Bcl-2 family members Mcl-1, Bcl-xl, Noxa, Bim, Bax, Bak and Bax (Supplementary Figure S4). As steady-state protein expression may vary significantly from mRNA levels, we additionally profiled each cell line by western blot (Figure 4). As expected, expression differed among the cell lines, particularly the levels of the $\mathrm{BH} 3-o n l y$ proteins Noxa and Bim as well as the anti-apoptotic proteins $\mathrm{Bcl}-2, \mathrm{Mcl}-1$ and $\mathrm{BCl}-\mathrm{xL}$. We found no significant correlation between $\mathrm{Mcl}-$ 1 sensitivity and protein expression for anti-apoptotic Mcl-1, $\mathrm{Bcl}-\mathrm{xL}$ and $\mathrm{Bcl}-2$ (Figures $5 \mathrm{a}$ and $\mathrm{c}$ ), nor for the expression of BH3-only proteins Bim and Noxa (Figures $5 \mathrm{~d}$ and e). Expression of pro-apoptotic executioner proteins Bak and
Bax weakly correlated with $\mathrm{Mcl}-1$ dependency, with an $R^{2}$ of 0.35 and 0.44 , respectively $(P<0.05)$ (Figures $5 f$ and $g$ ). Together, expression of single proteins at best only weakly correlates with $\mathrm{Mcl}-1$ dependency.

A combined index of $\mathrm{Bcl}-2$ family proteins better predicts Mcl-1 dependency. As Bcl-2 proteins work in concert to regulate apoptosis, a more holistic approach for predicting survival dependency on $\mathrm{Mcl}-1$ should include multiple protein expression levels. Using a multiple linear regression (1) where $y$ is cell viability after Mcl-1 knockdown and [protein] is the protein expression normalized to tubulin from the western blot analysis, we used a least-squares-fit of equation 1 to obtain the constant factors $b$ and $\mathrm{m}_{1-5}$. Mcl-1 and $\mathrm{Bcl}-\mathrm{xL}$ were included because of their role in singly or dually protecting the cell from apoptosis, as seen in Figures 1 and 2. Bak was chosen because of its mechanistic function to depolarize the mitochondria after $\mathrm{Mcl}-1$ and $\mathrm{Bcl}-\mathrm{xL}$ are antagonized and its significant correlation with $\mathrm{Mcl}-1$ sensitivity in Figure 5 . Bim and Noxa are important general and Mcl-1-specific antagonizing $\mathrm{BH} 3-$ only proteins, respectively.

$$
\begin{aligned}
\mathrm{y}= & \mathrm{b}+\mathrm{m}_{1} *[\mathrm{Mcl}-1]+\mathrm{m}_{2} *[\mathrm{Bcl}-\mathrm{xL}]+\mathrm{m}_{3} *[\mathrm{Noxa}]+\mathrm{m}_{4} \\
& *[\mathrm{Bim}]+\mathrm{m}_{5} *[\mathrm{Bak}] \\
\mathrm{y}= & 31.3+76.4 *[\mathrm{Mcl}-1]+89.9 *[\mathrm{Bcl}-\mathrm{xL}]+336[\mathrm{Noxa}] \\
& -104 *[\mathrm{Bim}]-230 *[\mathrm{Bak}]
\end{aligned}
$$


a

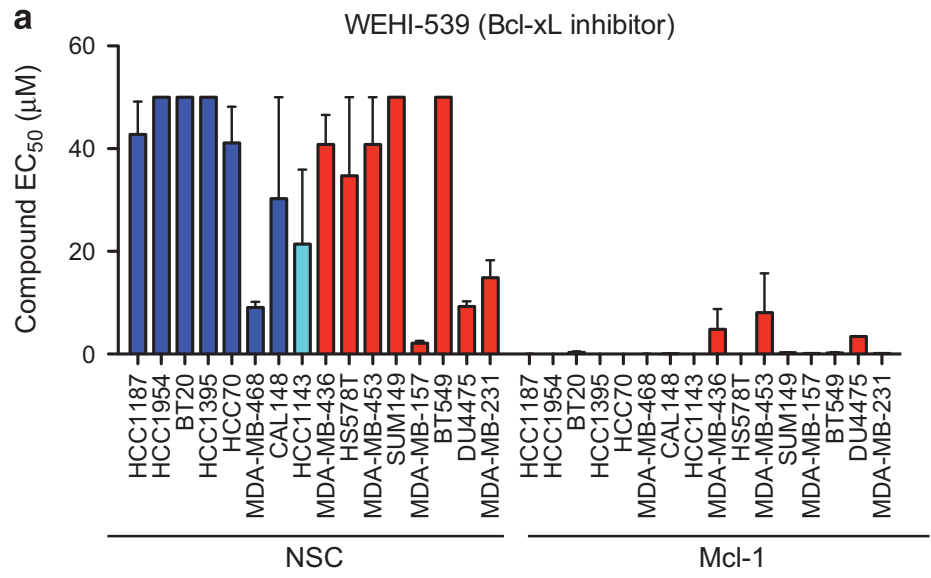

c

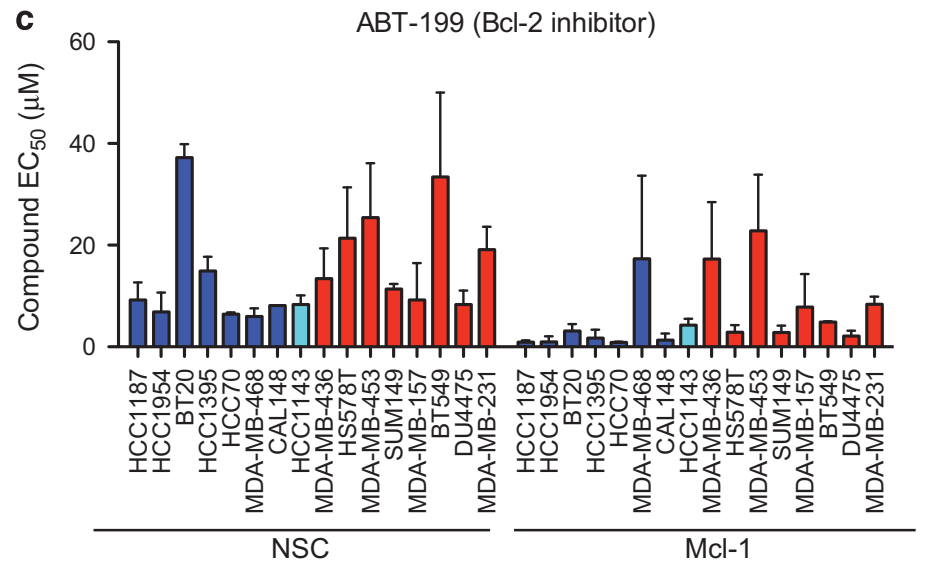

e $\quad$ ABT-263 (Bcl-xL \& Bcl-2 inhibitor)

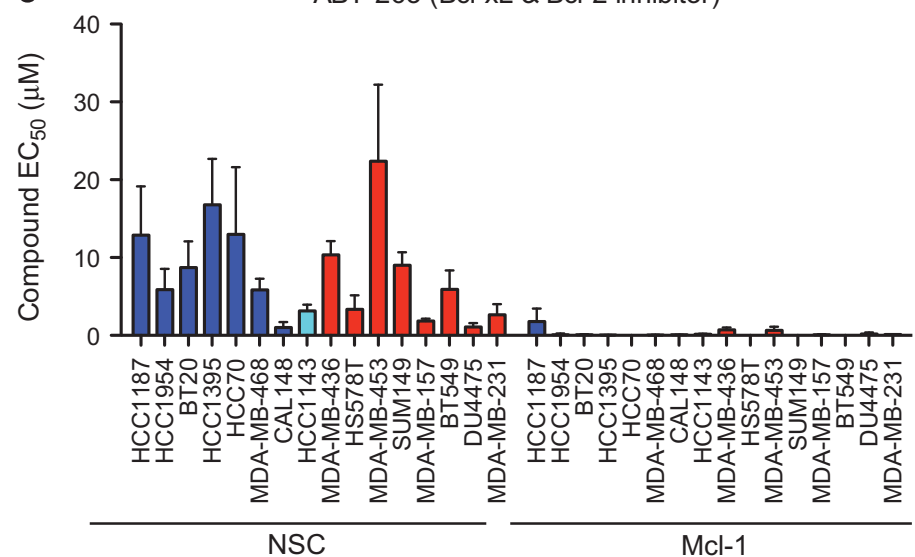

b

WEHI-539

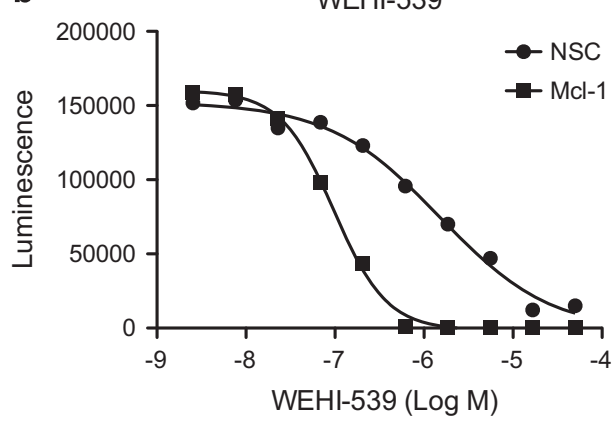

d

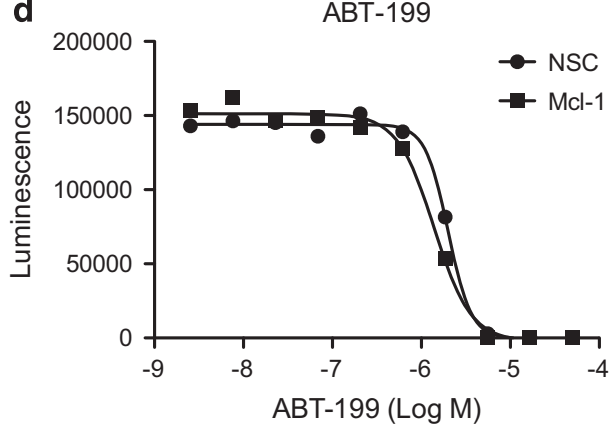

ABT-199

ABT-263

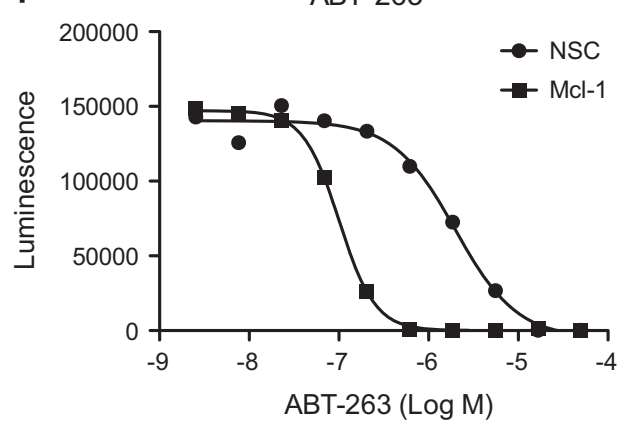

Figure $3 \mathrm{Bcl}-2$ and $\mathrm{Bcl}-\mathrm{xL}$ inhibitors in combination with Mcl-1 silencing. Cells were treated with NSC or Mcl-1 siRNA, then dosed with compound to measure IC ${ }_{50}$. (a) The observed IC ${ }_{50}$ of the selective Bcl-xL inhibitor WEHI-539 is reduced markedly across the panel when Mcl-1 is knocked out compared with NSC control. (b) Representative doseresponse curves in MDA-MB-157 cells treated with WEHI-539. In contrast, cells remained resistant to ABT-199 (a Bcl-2-selective inhibitor) irrespective of Mcl-1 knockdown across the panel (c) and exemplified in MDA-MB-157 cells (d). (e) Cell lines are also resistant to dual inhibition of Bcl-xL and Bcl-2 by the inhibitor ABT-263 when dosed with NSC siRNA, but co-dosing ABT-263 with Mcl-1 siRNA decreases compound IC ${ }_{50}$ similar to that observed with WEHI-539. Representative curves for MDA-MB-157 are shown in f. Shown are the mean of at least two independent experiments (error bars S.E.M.)

Using this equation, we predicted Mcl-1 sensitivity for each cell line and plotted it against the experimentally determined viability (Figure 6a). The resulting fit has an $R^{2}$ of 0.71 $(P<0.001)$. To identify the relative importance of each protein in the equation, we recalculated by singly removing each factor (Supplementary Figures S5 A-E). Removing Bak had the largest effect on $R^{2}$, followed by $\mathrm{Bcl}-\mathrm{xL}, \mathrm{Bim}, \mathrm{Mcl}-1$ and Noxa, respectively, which is consistent with our single-linear regression showing Bak as the strongest individual predictor of sensitivity among these five proteins. We further tested this 


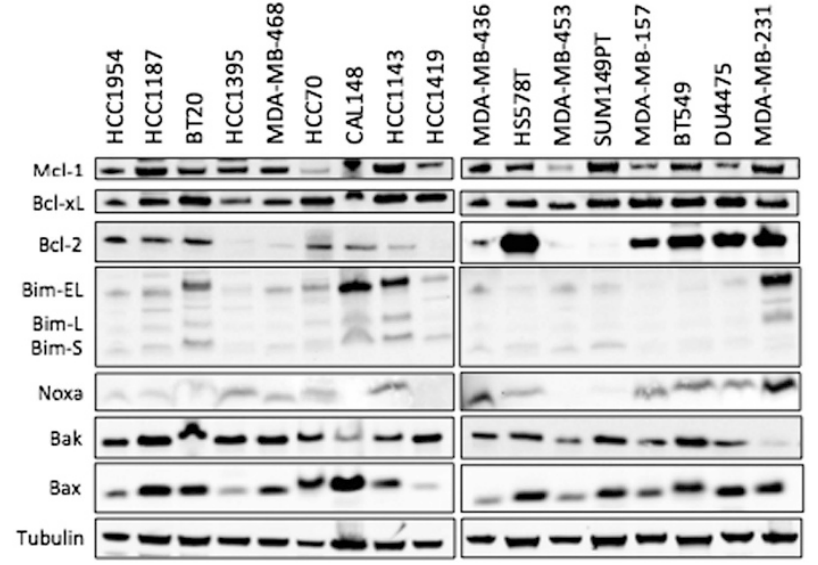

Figure $4 \quad B c l-2$ family protein expression levels in the TNBC cell line panel. Equal amount $(18 \mu \mathrm{g})$ of cell lysate was analyzed by western blot and probed for the indicated protein, with tubulin as a loading control equation by replacing the least significant contributor (Noxa) with $\mathrm{Bcl}-2$, but the quality of the fit decreased (Supplementary Figure S5F).

Coefficients depend on the protein's importance to predict viability and on the quantified western blot band intensity (e.g., the low-intensity Noxa bands result in a large coefficient). Therefore, this equation is highly dependent on uniform band staining between experiments, and necessitates a loading control to standardize results obtained under different conditions. Normalized mRNA expression is another metric that is often used as a surrogate for protein expression and is available for a large number of cancer cell lines in public databases (such as the CCLE), allowing rapid identification of more potentially sensitive cell lines for additional study. We tested whether mRNA levels predicted cell line sensitivity (Figure 6b). Although the goodness-of-fit and the slope both decreased, the overall $R^{2}$ was significant at $0.54(P<0.001)$ (Equation 3).

$$
\begin{aligned}
\mathrm{y}= & 675-52.5 *[\mathrm{Mcl}-1]+22.6 *[\mathrm{Bcl}-\mathrm{xL}]+6.27[\mathrm{Noxa}] \\
& +0.158 *[\mathrm{Bim}]-46.0 *[\mathrm{Bak}]
\end{aligned}
$$

a

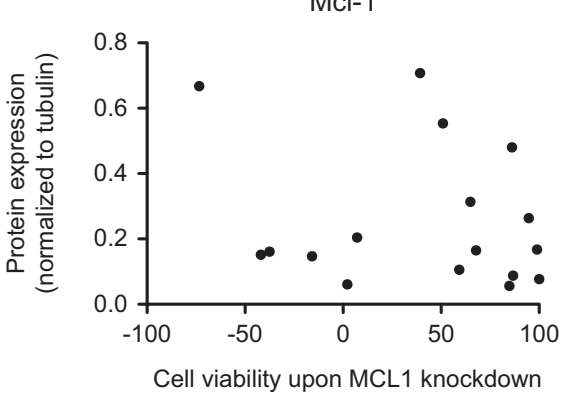

d

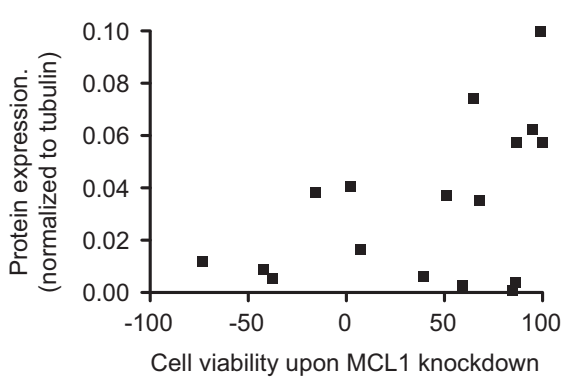

g

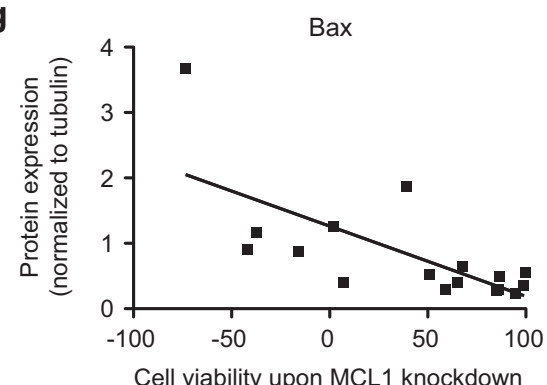

Bcl-xL

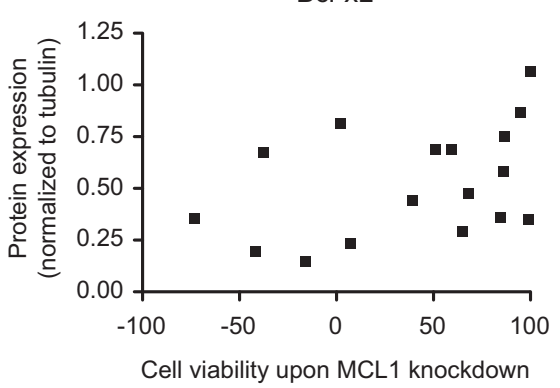

e

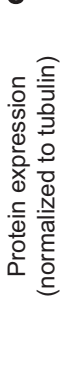

C

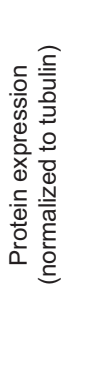

Bcl-2

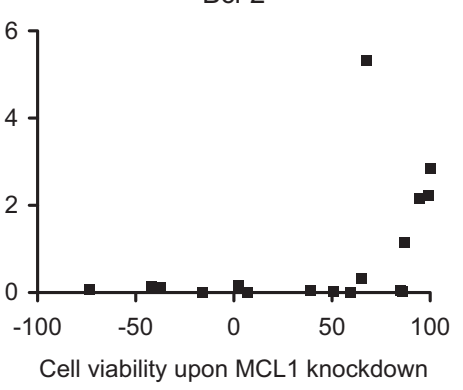

f

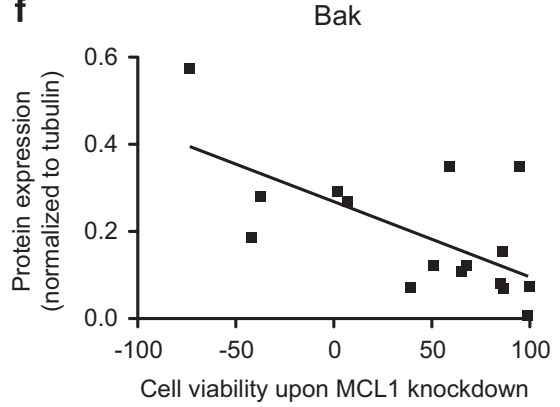

Figure 5 Individual Bcl-2 family protein expression does not strongly correlate with Mcl-1 sensitivity. (a-e) Tubulin-normalized protein expression levels for Mcl-1, Bcl-xL, Bcl-2, Noxa and Bim do not significantly correlate with cell viability after Mcl-1 knockdown. $(\mathbf{f}-\mathbf{g})$ Expression of executioner Bcl-2 family protein Bak and Bax correlates with Mcl-1 sensitivity with an $R^{2}$ of 0.34 and 0.44 , respectively $(P<0.05)$ 
a

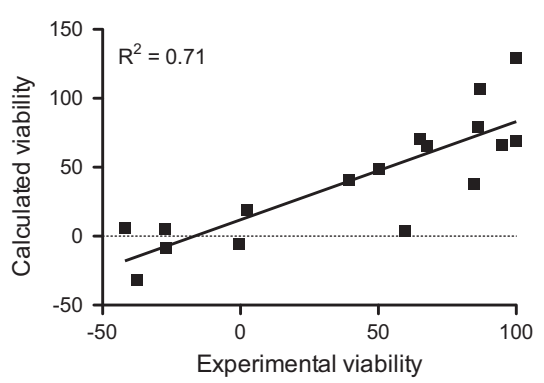

C

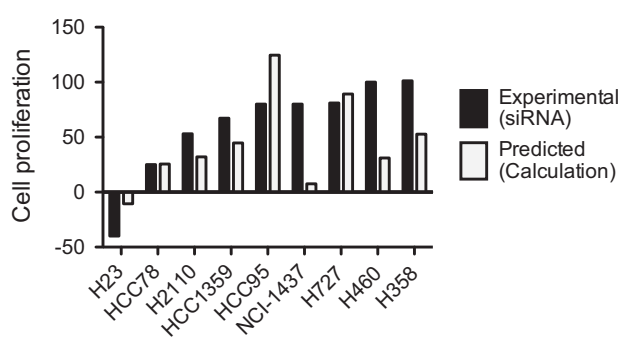

b

mRNA: MCL1; BCL2L1 (BCl-xL); BCL1L11 (Bim); BAK; PMAIP1 (Noxa)

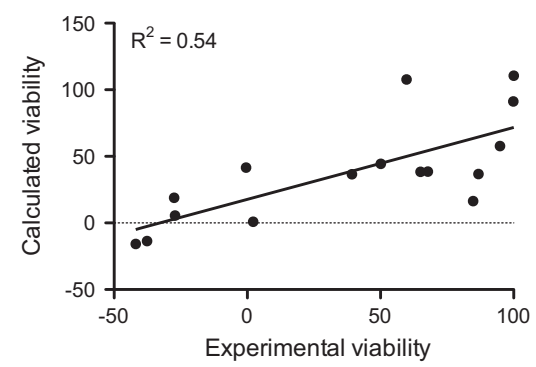

d

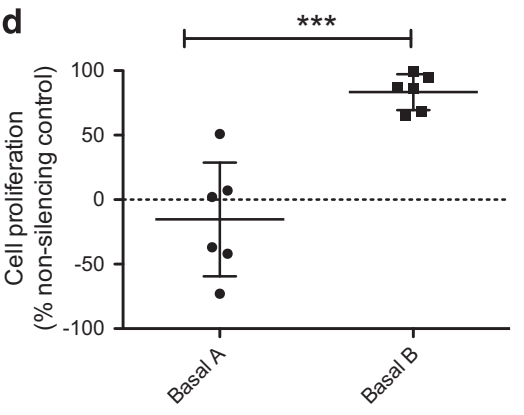

Figure 6 A combined index of multiple Bcl-2 family proteins predicts sensitivity to Mcl-1 knockdown. (a) A multiple linear regression was used to fit Mcl-1, Bcl-xL, Bim, Bak and Noxa protein expression to predict cell viability after Mcl-1 knockdown; the fit is plotted against experimental viability and a least-squares-fit linear regression line is drawn $\left(R^{2}=0.71, P<0.0001\right)$. (b) Using mRNA expression values results in a weaker, but still significant, overall fit $\left(R^{2}=0.54, P<0.001\right)$. (c) Using this mRNA-derived index, we predicted Mcl-1 sensitivity in a panel of nine non-small cell lung cancer cell lines, and compared those numbers with experimentally determined viability after silencing Mcl-1 expression. (d) Grouping cell lines by subtype indicates that Basal A significantly correlates with Mcl-1 sensitivity, whereas Basal B subtyped cell lines are Mcl-1 insensitive (twotailed $t$-test, $P<0.0004$, error bars S.D.)

We next tested whether the coefficients derived from TNBC cell lines would have predictive value in other cancer types. We silenced $\mathrm{Mcl}-1$ expression in a panel of non-small cell lung cancer cell lines and compared their experimental viability with predictions based on equation 3. The equation correctly forecasted the dependency of seven out of the nine tested, including identifying $\mathrm{H} 23$ and $\mathrm{HCC} 78$ as very sensitive and $\mathrm{H} 358$ and $\mathrm{H} 727$ as insensitive (Figure 6c). The equation incorrectly classified two cell lines as sensitive that the siRNA data revealed as insensitive.

Finally, we examined whether Mcl-1-sensitive cell lines segregate to a particular subtype of TNBC. In confirmation of a previous report, ${ }^{23}$ we found that $\mathrm{Mcl}-1$ sensitivity correlates with the Basal A TNBC subtype as defined by Neve et al., ${ }^{40}$ whereas the Basal B subtype is resistant (Figure 6d).

\section{Discussion}

Cancer cells often protect themselves from programmed cell death by upregulating anti-apoptotic members of the Bcl-2 protein family, or conversely, reducing expression of pro-death family members. Pharmacologically removing this apoptotic block can restore apoptosis and provides a useful therapeutic approach for treating tumors. ${ }^{38}$ The protein(s) responsible for apoptotic protection can differ for individual tumors and cell lines and have important implications on the choice of treatment. In this study, we found that a significant fraction of TNBC cell lines (41\%) are dependent upon Mcl-1 for survival, as evidenced by markedly reduced viability after $\mathrm{Mcl}-1$ protein knockdown, caspase 3/7 activation, Annexin $\mathrm{V}$ staining and mitochondrial depolarization by an Mcl-1-specific peptide. In contrast, silencing $\mathrm{Bcl}-\mathrm{xL}$ had only a modest effect in the TNBC cell lines examined. Strikingly, co-silencing both produced significantly increased cell death and reduced viability in the majority of cell lines, even those that were resistant to silencing either protein individually.

Consistent with these results, the Bcl-xL inhibitor WEHI-539 was largely ineffective when used alone against TNBC cells lines, but potently killed cells in combination with $\mathrm{Mcl}-1$ knockdown. Similarly, the dual $\mathrm{Bcl}-2$ and $\mathrm{Bcl}-\mathrm{xL}$ inhibitor ABT-263 was only mildly cytotoxic when used alone, indicating that $\mathrm{Bcl}-2$ and $\mathrm{Bcl}-\mathrm{xL}$ combined inhibition do not reduce viability, but in combination with $\mathrm{Mcl}-1$ silencing is strongly cytotoxic. These results suggest that inhibition of Mcl-1 in TNBC could resensitize tumors following acquired resistance to $\mathrm{Bcl}-2$ family chemotherapeutics, as is seen in other cancer types. ${ }^{26,27,41}$

We next sought to understand why some (but not all) cell lines responded to $\mathrm{Mcl}-1$ knockdown. Each cell line in this TNBC panel expresses executioner proteins Bak, Bax and $\mathrm{BH} 3-o n l y$ proteins, and their mitochondria depolarizes in response to $\mathrm{Bim}$. These data suggest that intrinsic pathway apoptotic blockade is due to antagonism by high levels of antiapoptotic proteins (e.g., $\mathrm{Mcl}-1, \mathrm{Bcl}-\mathrm{xL}$, etc). ${ }^{42}$ Although alternate routes exist to block apoptosis without affecting $\mathrm{Bcl}-2$ family proteins on the mitochondrion (such as by upregulating inhibitors of apoptosis proteins, or by blocking $\mathrm{BH} 3-$ only protein activation), the sensitivity of most cell lines to single $\mathrm{Mcl}-1$, or combined $\mathrm{Mcl}-1$ and $\mathrm{Bcl}-\mathrm{xL}$ knockdown, suggests that these other mechanisms are underutilized. We 
observed high (albeit varied) levels of $\mathrm{Mcl}-1$ and $\mathrm{Bcl}-\mathrm{xL}$ and low levels of $\mathrm{Bcl}-2$ protein expression across the TNBC cell line panel. Although expression of a particular anti-apoptotic protein does not necessarily indicate a cell is dependent on it for survival, ${ }^{13,14}$ significant expression is likely a necessary precondition for the protein to be an important sensor of cell death stimuli and thus a viable target for therapeutic manipulation. Indeed, all $\mathrm{Mcl}-1, \mathrm{Bcl}-\mathrm{xL}$ or dually sensitive cell lines expressed the requisite protein(s). In addition, Bak and Bax protein levels significantly (albeit weakly) correlated with reduced viability after $\mathrm{Mcl}-1$ knockdown, indicating their central importance to execute mitochondrial depolarization when anti-apoptotic Bcl-2 family antagonism is removed.

Cancer cells differ not just in the absolute expression levels of anti-apoptotic proteins, but also in their extent of pro-apoptotic protein activation. A cell line or tumor with higher pro-apoptotic activation is more 'primed for death' and will require more anti-apoptotic proteins to survive. ${ }^{42}$ Therefore, it is not surprising that we saw no correlation of $\mathrm{Mcl}-1$ protein or mRNA with $\mathrm{Mcl}-1$ dependency because these fail to account for differences in cellular pro-apoptotic potential. Similarly, the extent of unbound anti-apoptotic proteins is also an important criteria to predict dependency. Over $40 \%$ of tested lines responded to $\mathrm{Mcl}-1$ knockdown alone, but most of the insensitive lines required combined $\mathrm{Mcl}-1$ and $\mathrm{Bcl}-\mathrm{xL}$ knockdown to die, suggesting that these lines express additional anti-apoptotic proteins sufficient to sequester any released pro-apoptotic factors after $\mathrm{Mcl}-1$ loss. ${ }^{14}$

Interestingly, knockdown of $\mathrm{Bcl}-\mathrm{xL}$ alone had relatively little effect compared with $\mathrm{Mcl}-1$ knockdown. Although the two proteins have unique specificities for $\mathrm{BH}$-only proteins (e.g., Mcl-1 can bind Noxa but Bcl-xL can bind Bad), their overall anti-apoptotic mechanisms are similar. This discrepancy has been observed in other cancer types as well, including acute myeloid leukemia ${ }^{43}$ and ER-positive breast cancer. $^{12}$ The Bad-BH3 peptide, which antagonizes $\mathrm{Bcl}-2$, $\mathrm{Bcl}-\mathrm{w}$ and $\mathrm{Bcl}-\mathrm{xL}$, depolarized mitochondria to a lesser extent than the $\mathrm{Mcl}-1$-specific peptide. This suggests that in these cell lines the pool of $\mathrm{Bcl}-2, \mathrm{Bcl}-\mathrm{w}$ and $\mathrm{Bcl}-\mathrm{xL}$ contributes marginally compared with $\mathrm{Mcl}-1$ in protection against apoptotic induction. Perhaps $\mathrm{Mcl}-1$ more actively sequesters pro-apoptotic proteins, with the bulk of Bcl-xL prevented from blocking apoptosis by localization or inactivation.

As a result of these complications, we are unsurprised to find several previously published multi-protein indexes for predicting response to Bcl-2 family inhibitors. ${ }^{17,44}$ Our data suggest that an additional metric for predicting dependency on a particular anti-apoptotic protein will consider the relative amounts of both pro- and anti-apoptotic proteins. We found that an index of five proteins (Mcl-1, Bcl-xL, Bim, Bak and Noxa) could highly predict $\mathrm{Mcl}-1$ sensitivity with an $R^{2}$ of 0.71 . As these predictions were based on in vitro experiments, their performance in vivo with complicating factors such as stroma and the associated microenvironment deserves further study.

Including $\mathrm{Bcl}-2$ in the index did not improve our viability predictions in TNBC. The TNBC cell lines tested cells were resistant to the $\mathrm{Bcl}-2$-specific inhibitor ABT-199 alone or in combination with $\mathrm{Mcl}-1$ silencing, which suggests that $\mathrm{Bcl}-2$ provides little anti-apoptotic protection in these cell lines. However, including $\mathrm{Bcl}-2$ may make the equation more broadly applicable to other cancer types that do heavily rely on $\mathrm{Bcl}-2$ to protect against apoptosis during Mcl-1 loss.

Our results suggest that a $\mathrm{BH} 3-$ mimetic capable of inhibiting Mcl-1 interactions may prove efficacious as a single-agent therapeutic in some TNBC tumors; whereas single-agent inhibition of $\mathrm{Bcl}-2$ or Bcl-xL may be largely ineffective. In other TNBC tumors, combination therapy may be required. The highly significant correlation of Basal A subtype status to $\mathrm{Mcl}-1$ dependency in these cell lines should be further investigated using patient-derived samples, and could prove to be an effective biomarker for clinical breast cancer patient selection. In other cancer types, an analysis of the protein or mRNA levels of Bcl-2 family members (e.g., Mcl-1, Bcl-xL, Bak and Bim) may be useful in a multi-factorial expression as shown here to mechanistically predict which tumors are likely to respond to an $\mathrm{Mcl}-1$ inhibitor across different tumor types.

\section{Materials and Methods}

Cell lines and culture conditions. Cell lines were obtained from the American Type Culture Collection (ATCC, Manassas, VA, USA) except for Cal148 (obtained Deutsche Sammlung von Mikroorganismen und Zellkulturen $\mathrm{GmbH}$, Braunschweig, Germany) and Sum149PT (obtained from Asterand, Detroit, Ml, USA). Cells were cultured in recommended media.

Protein knockdown and cell viability. Protein expression was silenced using siRNA. On-targetPLUS motifs specific to the 3'-UTR or ORF for Mcl-1 and Bcl-xL were obtained from Dharmacon (Thermo Scientific, Waltham, MA, USA). Cells were plated in antibiotic-free medium at 5000 cells per well in a 96-well plate. After $24 \mathrm{~h}$, medium was replaced with siRNA-containing medium consisting of $0.01 \%$ Dharmafect I transfection reagent and $25 \mathrm{nM}$ siRNA sequence specific to the target or NSC motif. Control wells received normal media. Cell viability was measured after 5 days using Cell Titer-Glo (Promega, Fitchburg, WI, USA), a fluorescent assay that produces signal corresponding to ATP concentration (a proxy for cell count). Signal was measured on the Spectramax M5 plate reader (Molecular Devices, Sunnyvale, CA, USA) averaged over five replicate wells. Conditions were optimized such that cell viability was reduced by the NSC siRNA by no $>30 \%$, and protein knockdown was verified by western blot analysis using lysates collected at the conclusion of the experiment.

Flow cytometry. Cells were plated in a six-well dish at 100000 cells per well. Non-silencing or Mcl-1 siRNA were dosed for $24 \mathrm{~h}$ before collection and staining with fluorescein isothiocyanate conjugates of annexin $\mathrm{V}$ and propidium iodide (Life Technologies, Grand Island, NY, USA) and analyzed on a 5 Laser LSRII (BD Biosciences, San Jose, CA, USA)

Western blotting. Cell lysates were collected in RIPA buffer. Equal amount of lysate $(18 \mu \mathrm{g})$ was boiled in SDS protein loading buffer supplemented with $10 \mathrm{mM}$ DTT, separated by SDS-PAGE, transferred to Immobilon-FL PVDF membrane (Millipore, Billerica, MA, USA), and blotted for indicated protein. Antibodies for Mcl-1 (Y37), Bcl-xL (E18) obtained from Abcam (Cambridge, UK); Bcl-2 (50E3), Bak and Bax from Cell Signaling Technologies (Danvers, MA, USA); Bim (H-191) and Bad (C-7) from Santa Cruz Biotechnology (Dallas, TX, USA); Noxa and $\alpha$-tubulin (DM1A) from Millipore. Each was used at a 1:1000 dilution. Blots were probed with species-appropriate IRDye-conjugated secondary antibodies (Licor, Lincoln, NE, USA) at 1:20000 dilution and measured on the Licor Odyssey system. Quantification performed on the Licor Image Studio.

BH3 profiling. Synthetic peptides were ordered from Genscript (Piscataway, NJ, USA). Sequences are as previously described for MS- $1,{ }^{26} \mathrm{Bim}-\mathrm{BH} 3$ and Bad-BH3 $3{ }^{27}$ $\mathrm{BH} 3$ profiling was conducted and analyzed as described. ${ }^{27}$ Fluorescence was measured on a Biotek Cytation 3 (Winooski, VT, USA) after $1.5 \mathrm{~h}$. DMSO control was used for zero depolarization, and FCCP at $20 \mu \mathrm{M}$ was used for $100 \%$ depolarization. Normalized percent polarization is calculated as (Sample-FCCP)/(DMSO-FCCP).

Compound studies. Cells were plated in antibiotic-free medium to a final count of 1000 cells per well in a 96-well plate and left for $24 \mathrm{~h}$. NSC or Mcl-1 siRNA were added as described above. After 2 days, compounds ABT-263, ABT-199 or 
WEHI-539 (SelleckChem, Houston, TX, USA) were added in 10-point, threefold serial dilutions to a final DMSO concentration of $0.5 \%$. Cell viability was quantified on day 5 by Cell Titer-Glo (Promega, Madison, WI, USA) and read on the Spectramax M5 plate reader (Molecular Devices). Results shown are the average of two or more independent experiments.

mRNA expression data. Robust multi-array average-normalized mRNA expression data for each cell line (excluding Sum149, for which data were not available) was obtained from the Broad-Novartis Cancer Cell Line Encyclopedia (CCLE), available at http://www.broadinstitute.org/ccle.

Regression analysis. Linear regression and statistical analysis was performed in Prism (Graphpad Software, La Jolla, CA, USA). Multiple linear regression was performed using the LINEST function in Microsoft Excel 2010 (Redmond, WA, USA).

\section{Conflict of Interest}

The authors declare no conflict of interest.

Acknowledgements. This research was supported by the National Cancer Institute Breast Specialized Programs of Research Excellence (SPORE) grant at Vanderbilt University (grant number 2P50CA098131-11). Flow cytometry experiments were performed in the Vanderbilt Medical Center (VMC) Flow Cytometry Shared Resource. The VMC Flow Cytometry Shared Resource is supported by the Vanderbilt-Ingram Cancer Center (P30 CA68485) and the Vanderbilt Digestive Disease Research Center (DK058404).

1. Carey LA. Directed therapy of subtypes of triple-negative breast cancer. Oncologist 2011; 16: 71-78.

2. Schmadeka R, Harmon BE, Singh M. Triple-negative breast carcinoma. Am J Clin Pathol 2014; 141: 462-477.

3. Hudis CA, Gianni L. Triple-negative breast cancer: an unmet medical need. Oncologist 2011; 16: 1-11.

4. Dent R, Trudeau M, Pritchard KI, Hanna WM, Kahn HK, Sawka CA et al. Triple-negative breast cancer: clinical features and patterns of recurrence. Clin Cancer Res 2007; 13: 4429-4434.

5. McCarthy N, Mitchell G, Bilous M, Wilcken N, Lindeman GJ. Triple-negative breast cancer: making the most of a misnomer. Asia Pac J Clin Oncol 2012; 8: 145-155.

6. O'Toole SA, Beith JM, Millar EKA, West R, McLean A, Cazet A et al. Therapeutic targets in triple negative breast cancer. J Clin Pathol 2013; 66: 530-542.

7. Youle RJ, Strasser A. The BCL-2 protein family: opposing activities that mediate cell death. Nat Rev Mol Cell Biol 2008; 9: 47-59.

8. Czabotar PE, Lessene G, Strasser A, Adams JM. Control of apoptosis by the BCL-2 protein family: implications for physiology and therapy. Nat Rev Mol Cell Biol 2014; 15: 49-63.

9. Billard C. BH3 mimetics status of the field and new developments. Mol Cancer Ther 2013; 12: $1691-1700$.

10. Gandhi L, Camidge DR, Ribeiro de Oliveira M, Bonomi P, Gandara D, Khaira D et al. Phase I study of Navitoclax (ABT-263), a novel Bcl-2 family inhibitor, in patients with small-cell lung cancer and other solid tumors. J Clin Oncol 2011; 29: 909-916.

11. Vogler M, Dinsdale D, Dyer MJS, Cohen GM. Bcl-2 inhibitors: small molecules with a big impact on cancer therapy. Cell Death Differ 2009; 16: 360-367.

12. Vaillant F, Merino D, Lee L, Breslin K, Pal B, Ritchie ME et al. Targeting BCL-2 with the BH3 mimetic ABT-199 in estrogen receptor-positive breast cancer. Cancer Cell 2013; 24: 120-129.

13. Goldsmith KC, Lestini BJ, Gross M, Ip L, Bhumbla A, Zhang X et al. BH3 response profiles from neuroblastoma mitochondria predict activity of small molecule $\mathrm{Bcl}-2$ family antagonists. Cell Death Differ 2010; 17: 872-882.

14. Morales AA, Kurtoglu M, Matulis SM, Liu J, Siefker D, Gutman DM et al. Distribution of Bim determines Mcl-1 dependence or codependence with $\mathrm{Bcl}-\mathrm{x} \mathrm{L} / \mathrm{Bcl}-2$ in Mcl-1-expressing myeloma cells. Blood 2011; 118: 1329-1339.

15. Chen L, Willis SN, Wei A, Smith BJ, Fletcher Jl, Hinds MG. Differential targeting of prosurvival Bcl-2 proteins by their BH3-only ligands allows complementary apoptotic function. Mol Cell 2005; 17: 393-403.

16. Thomas LW, Lam C, Edward SW. Mcl-1; the molecular regulation of protein function. FEBS Lett 2010; 584: 2981-2989.

17. Zhang H, Guttikonda S, Roberts L, Uziel T, Semizarov D, Elmore SW et al. Mcl-1 is critical for survival in a subgroup of non-small-cell lung cancer cell lines. Oncogene 2011; 30: 1963-1968.

18. Zhang Z, Liu Y, Song T, Xue Z, Shen X, Liang F et al. An antiapoptotic Bcl-2 family protein index predicts the response of leukaemic cells to the pan-Bcl-2 inhibitor S1. Br J Cancer. 2013; 108: 1870-1878.

19. Wei G, Margolin AA, Haery L, Brown E, Cucolo L, Julian B et al. Chemical genomics identifies small-molecule $M C L 1$ repressors and $B C L-x L$ as a predictor of $M C L 1$ dependency. Cancer Cell 2012; 21: 547-562.
20. Beroukhim R, Mermel $\mathrm{CH}$, Porter D, Wei G, Raychaudhuri S, Donovan $\mathrm{J}$ et al. The landscape of somatic copy-number alteration across human cancers. Nature 2010; 463: 899-905.

21. Ding Q, He X, Xia W, Hsu JM, Chen CT, Li LY et al. Myeloid cell leukemia-1 inversely correlates with glycogen synthase kinase-3beta activity and associates with poor prognosis in human breast cancer. Cancer Res 2007; 67: 4564-4567.

22. Mitchell C, Yacoub A, Hossein H, Martin AP, Bareford MD, Eulitt P et al. Inhibition of MCL-1 in breast cancer cells promotes cell death in vitro and in vivo. Cancer Biol Ther 2010; 10: 903-917.

23. Petrocca F, Altschuler G, Tan SM, Mendillo ML, Yan H, Jerry DJ et al. A genome-wide siRNA screen identifies proteasome addiction as a vulnerability of basal-like triple-negative breast cancer cells. Cancer Cell 2013; 24: 182-196.

24. Balko JM, Giltname JM, Wang K, Schwarz LJ, Young CD, Cook RS et al. Molecular profiling of the residual disease of triple-negative breast cancers after neoadjuvant chemotherapy identifies actionable therapeutic targets. Cancer Discov 2014; 4: 232-245.

25. Wertz IE, Kusam S, Lam C, Okamoto T, Sandoval W, Anderson DJ et al. Sensitivity to antitubulin chemotherapeutics is regulated by MCL1 and FBW7. Nature 2011; 471: $110-114$.

26. van Delft MF, Wei AH, Mason KD, Vandenberg CJ, Chen L, Czabotar PE et al. The BH3 mimetic ABT-737 targets selective Bcl-2 proteins and efficiently induces apoptosis via Bak/ Bax if Mcl-1 is neutralized. Cancer Cell 2006; 10: 389-399.

27. Tahir SK, Yang X, Anderson MG, Morgan-Lappe SE, Sarthy AV, Chen J et al. Influence of Bcl-2 family members on the cellular response of small-cell lung cancer cell lines to ABT-737. Cancer Res. 2007; 67: 1176-1183.

28. Rooswinkel RW, van de Kooij B, Verheij M, Borst J. Bcl-2 is a better ABT-737 target than $\mathrm{Bcl}-\mathrm{xL}$ or $\mathrm{Bcl}-\mathrm{w}$ and only Noxa overcomes resistance mediated by $\mathrm{Mcl}-1, \mathrm{Bfl}-1$, or $\mathrm{Bcl}-\mathrm{B}$. Cell Death Dis 2012; 3: e366.

29. Zhou P, Levy NB, Xie H, Qian L, Lee CY, Gascoyne RD et al. MCL1 transgenic mice exhibit a high incidence of B-cell lymphoma manifested as a spectrum of histologic subtypes. Blood 2001; 97: 3902-3909.

30. Moulding DA, Giles RV, Spiller DG, White MR, Tidd DM, Edwards SW. Apoptosis is rapidly triggered by antisense depletion of MCL-1 in differentiating U937 cells. Blood 2000; 96 : 1756-1763.

31. Chetoui N, Sylla K, Gagnon-Houde JV, Alcaide-Loridan C, Charron D, Al-Daccak R et al. Down-regulation of $\mathrm{Mcl}-1$ by small interfering RNA sensitizes resistant melanoma cells to Fas-mediated apoptosis. Mol Cancer Res 2008; 6: 42-52.

32. Heere-Ress E, Thallinger C, Lucas T, Schlagbauer-Wadl H, Wacheck V, Monia BP et al. Bcl$\mathrm{X}(\mathrm{L})$ is a chemoresistance factor in human melanoma cells that can be inhibited by antisense therapy. Int J Cancer 2002; 99: 29-34.

33. Colak S, Zimberlin CD, Fessler E, Hogdal L, Prasetyanti PR, Grandela CM et al. Decreased mitochondrial priming determines chemoresistance of colon cancer stem cells. Cell Death Differ 2014; 21: 1170-1177.

34. Ryan J, Letai A. BH3 profiling in whole cells by fluorimeter or FACS. Methods 2013; 61 156-164.

35. Certo M, Del Gaizo Moore V, Nishino M, Wei G, Korsmeyer S, Armstrong SA, Letai A. Mitochondria primed by death signals determine cellular addiction to antiapoptotic BCL-2 family members. Cancer Cell 2006; 9: 351-365.

36. Foight GW, Ryan JA, Gullá SV, Letai A, Keating AE. Designed BH3 peptides with high affinity and specificity for targeting Mcl-1 in cells. ACS Chem Biol. 2014; 9: 1962-1968.

37. Lessene G, Czabotar PE, Sleebs BE, Zobel K, Lowes KN, Adams JM. Structure-guided design of a selective BCL-X(L) inhibitor. Nat Chem Biol 2013; 9: 390-397.

38. Tse C, Shoemaker AR, Adickes J, Anderson MG, Chen J, Jin S. ABT-263: a potent and orally bioavailable Bcl-2 family inhibitor. Cancer Res 2008; 68: 3421-3428.

39. Souers AJ, Leverson JD, Boghaert ER, Ackler SL, Catron ND, Chen J et al. ABT-199, a potent and selective BCL-2 inhibitor, achieves antitumor activity while sparing platelets. Nat Med 2013; 19: 202-208.

40. Neve RM, Chin K, Fridlyand J, Yeh J, Baehner FL, Fevr T. A collection of breast cancer cell lines for the study of functionally distinct cancer subtypes. Cancer Cell 2006; 10: 515-527.

41. Tromp JM, Geest CR, Breij EC, Elias JA, van Laar J, Luijks DM. Tipping the NOXA/Mcl-1 balance overcomes ABT-737 resistance in chronic lymphocytic leukemia. Clin Cancer Res 2012; 18: 487-498.

42. Deng J, Carlson N, Takeyama K, Dal Cin P, Shipp M, Letai A. BH3 profiling identifies three distinct classes of apoptotic blocks to predict response to ABT-737 and conventional chemotherapeutic agents. Cancer Cell 2007; 12: 171-185.

43. Glaser SP, Lee EF, Trounson E, Bouillet P, Wei A, Fairlie WD et al. Anti-apoptotic Mcl-1 is essential for the development and sustained growth of acute myeloid leukemia. Genes Dev 2012; 26: 120-125.

44. Al-Harbi S, Hill BT, Mazumder S, Singh K, Devecchio J, Choudhary G. An antiapoptotic $\mathrm{BCL}-2$ family expression index predicts the response of chronic lymphocytic leukemia to ABT-737. Blood 2011; 118: 3579-3590. 